\title{
An Accurate PSO-GA Based Neural Network to Model Growth of Carbon Nanotubes
}

\author{
Mohsen Asadnia, ${ }^{1}$ Amir Mahyar Khorasani, ${ }^{2}$ and Majid Ebrahimi Warkiani ${ }^{3}$ \\ ${ }^{1}$ Department of Engineering, Macquarie University, Sydney, NSW 2109, Australia \\ ${ }^{2}$ School of Engineering, Deakin University, Waurn Ponds, VIC 3125, Australia \\ ${ }^{3}$ School of Biomedical Engineering, University of Technology, Sydney, NSW 2007, Australia \\ Correspondence should be addressed to Mohsen Asadnia; mohsen.asadnia@mq.edu.au
}

Received 12 April 2017; Revised 10 July 2017; Accepted 25 July 2017; Published 6 September 2017

Academic Editor: Yasuhiko Hayashi

Copyright (C) 2017 Mohsen Asadnia et al. This is an open access article distributed under the Creative Commons Attribution License, which permits unrestricted use, distribution, and reproduction in any medium, provided the original work is properly cited.

By combining particle swarm optimization (PSO) and genetic algorithms (GA) this paper offers an innovative algorithm to train artificial neural networks (ANNs) for the purpose of calculating the experimental growth parameters of CNTs. The paper explores experimentally obtaining data to train ANNs, as a method to reduce simulation time while ensuring the precision of formal physics models. The results are compared with conventional particle swarm optimization based neural network (CPSONN) and Levenberg-Marquardt (LM) techniques. The results show that PSOGANN can be successfully utilized for modeling the experimental parameters that are critical for the growth of CNTs.

\section{Introduction}

Increasing requirements for high manufacturing efficiency such as low throughput time, better product quality, and cheaper finished parts are still driving the equipment manufacturers and the fabrication industries in their search for new technologies. The increasing requirements drive the industry to search for smaller transistors, where size reductions result in higher clock frequencies and lower power dissipation. CNTs show promise in satisfying the need for smaller transistors as a result of their physical and electrical properties $[1,2]$. The mechanisms involved in growing CNTs are often complex with numerous experimental parameters that need precise control and often the growth-rate is very slow. Moreover, the growth process involves precursor materials, hydrocarbons, carrier gases, expensive equipment, and high thermal budget. Generating a simulator platform that allows us to optimize the growth parameters before conducting trial and error optimization through experiments will greatly save time and money. Therefore, in this paper, we present a novel algorithm by combining both particle swarm optimization (PSO) and genetic algorithm (GA) which predicts the experimental growth results of CNT and allows making parameter optimization less cumbersome. Ton et al. [3] have presented a numerical piecewise nonlinear approximation of the nonequilibrium mobile charge density to be used in the modeling of CNT transistors. Similarly, Yamacli and Avci [4] have developed a parameterisable model of CNTFET nanoelectronics. Recent advances in ANNs have been made possible through the efforts to model and simulate the behaviour of CNTS. Through the imitation of biological systems and analysis of brain structures these ANNs have developed human-like performance [3-6].

However, ANNs used for pattern classification and optimization problems often suffered from issues such as finding appropriated architecture to perform the satisfactory modeling performance [7]. Considerable research has been conducted in the development of new architectures and learning algorithms of the neural networks to achieve this objective, such as modular neural networks [8], hybrid neural networks [9], and evolutionary algorithms and evolutionary programming [10]. 
Seeking improved performance of conventional neural networks, researchers $[11,12]$ have turned to GA. Three evolutionary operations are required to implement a GA: selection, crossover, and mutation. It has been found in experiments that, with large training samples, the convergence speed for the GA would be significantly reduced [13]. Furthermore, application of the crossover operation in GA to neural networks can result in what is known as the "permutation problem." Consequently, the employment of GA is seen as a generally complicated process. Recently, other evolutionary techniques such as PSO have been applied in other branches of engineering $[14,15]$. By contrast, the PSO algorithm does not have two of the evolutionary operators of GA (crossover and mutation). The reduction in parameters yields a faster convergence which is easier to implement [16]. Accordingly, PSOs are suitable for approaching dynamic or problems that change rapidly over time [17].

The superiority of PSO and GA to the BP algorithm stems from the ability of PSO and GA trained ANNs to deal with nondifferentiable functions and work without gradient information. But one of the most notorious problems with the application of crossover to neural networks is known as falling in the local minimums and failing to converge. This problem is more visible when the number of data sets is not enough. Another common problem in conventional NNs is overfitting. If the number of weights of NN exceeds the number of data sets for the training of $\mathrm{NN}$ to some extent, "overfitting" may occur. GA are capable of isolating global optimums and, however, converge at low speed to this optimum. On the other hand, PSOs converge quickly albeit at a greater risk of isolating and being trapped at a local optimum. The optimization problem that arises from attempts to balance these two techniques can be solved by particle swarm intelligence. In order to overcome the downsides associated with each algorithm a combination of GA and PSO may be used which is referred to as GAPSO.

In GA, the binary strings of the initial population are generated randomly, so different runs of GA often give similar results. The idea of PSOGANN is to select these initial populations appropriately by using PSONN.

As mentioned before, in the case of lack of enough training data for network, neither conventional PSO nor GA based NN can provide a proper learning method for training NNs. Having a robust algorithm that could be trained with less training data could be a promising method for the application of NNs in microelectromechanical system (MEMS) fabrication and many other engineering fields.

In this study, a novel PSO-GA based neural network is proposed for improving the training capacity of neural network. To evaluate the performance of the proposed PSO algorithm, the training capacity of improved PSO-based ANN is first tested and then compared to that of a conventional PSObased ANN and a back propagation-based ANN, using the experimental data obtained from carbon nanotube growth process. Optimal back propagation-based neural network architecture is designed using MATLAB Neural Network Toolbox. Programs of PSOGANN and conventional PSObased ANN are accomplished in $\mathrm{C}++$.

\section{Genetic Algorithm}

A GA emulates the evolutionary characteristic of survival of the fittest. At each phase, encoded chromosomes are simulated; the algorithm establishes the strength of each chromosome. The chromosomes mutate with crossover producing the next generation. Then the process repeats. The input parameters for GA are a set of solutions (the chromosomes of the GA) and a fitness function defining success characteristics and stopping criteria. At each step of the algorithm chromosomes are first evaluated for suitability against the success characteristics. Subsequently successful chromosomes are randomly pooled to mate. Pairs of chromosomes in this pool randomly share genetic information with each other. The chromosomes are evaluated against the stopping criteria. Then the process repeats if the criteria are not met. These features of GA make it adequate for handling large, nonlinear problems with unpredictable results. Relying on multipoint search and algorithmic features, the chance of convergence to the universal optimal solution is much higher than the chance of falling into a local optimal solution. GA has a positive track record successfully having dealt with problems in a variety of fields, including but not limited to optimization, fuzzy logic, $\mathrm{NN}$, expert systems, and scheduling [11].

\section{IPSOGA Based ANN}

Particle swarm optimization is a population based stochastic optimization algorithm. In PSO algorithm the potential solutions, called particles, including weights and thresholds vectors fly through the problem space by following the current optimal particles. During training, after initializing PSO parameters using a group of random particles (solutions), optimal solution is achieved through the solution space [9]. Therefore, the velocity and position of the obtained optimal solution are updated according to its own experience and global cooperation. Despite regular PSOs converging rapidly on solutions, they may often become trapped within local maxima and minima [12]. To obviate this problem and improve its training capacity, a developed PSO algorithm is proposed. An improvement to the PSO algorithm is proposed by considering best and worst case particle positions. By nature of the algorithm best case positions will gravitate towards the optimal positions, away from worst case positions $[13,18]$.

Individual particles in the swarm are represented by $n$ dimensional position and velocity vectors contained in $S$, the search space:

$$
\begin{aligned}
X i & =[x i 1, x i 2, \ldots, x i n] T E S \\
V i & =[v i 1, v i 2, \ldots, v i n] T E S
\end{aligned}
$$

The evaluation of each particle is performed against the success function, with individual best positions being cumulatively stored in a position vector:

$$
P i=[P i 1, P i 2, \ldots, P i n] T \text {. }
$$

A global optimum position, $\mathrm{Pg}$, is established from an evaluation of individual positions. 
Between each iteration the new velocity of each particle is calculated from the distance to the global best position, the local best position, and an inertia weight from the previous velocity:

$$
\begin{aligned}
V(t+1)= & w * V(t)+c 1 r 1(P i(t)-X i(t)) \\
& +c 2 r 2(P g(t)-X i(t))
\end{aligned}
$$

where $c n * r n$ yields a randomly distributed acceleration coefficient.

The updated position can be given by taking the sum of the previous position and current velocity over the next iteration:

$$
X(t+1)=X(t)+V(t+1)
$$

The proposed algorithm (PSOGANN) is developed by taking advantage of both PSO and GA into the training process. A stopping criterion (which can be either the maximum number of iterations or reaching to a certain MSE) is imposed and if the PSO is unable to meet the stop criteria, the best population for GA (including weights and biases) is determined by PSO and GA will again search for the best parameter set. This process will continue until the stop criterion is satisfied. Figure 1 shows the flow chart of the proposed algorithm and details are presented below.

Step 1. Initialization of PSOGANN parameters: This includes (a) determination of the initial PSONN parameters $(c 1, c 2$, $c 3, w, V_{\max }$, and $\left.V_{\min }\right)$; (b) select weights and biases for the network randomly (first iteration); (c) selecting initial position and velocity vectors for all the particles (randomly); (d) selecting initial values of $P_{g}(t)$ and $P_{i}(t)$ randomly; (e) determination of number of circuits of group 1, which is number of generations where PSO can try to meet the stopping criteria in each step before its current best particle $\left(P_{g}(t)\right)$ is saved as one of the GA's populations; (f) number of circuits of group 2, which is number of initial populations in GA.

Step 2. Compute fitness of individual particles by the feedforward network.

Step 3. (a) Perform PSO operators to find the best PSONN parameters. (b) Update weights and thresholds according to equations (1) and (2) until "counter $1>$ number of circuits of group 1" is satisfied.

Step 4. (a) Best position of PSO algorithm saves as an initial population for GA; (b) counter 1 resets; (c) algorithm continues to search for the optimal PSO parameters for the current set of network weights and biases until the tolerance is met in Step 3.

Step 5. (a) If the tolerance is not met after the maximum number (counter 2), perform GA algorithm by initial populations which are saved by PSO in previous steps. (b) Continue until stop criterion is met in Step 4.

\section{Carbon Nanotube Growth Process}

4.1. Sample Preparation. A $4^{\prime \prime}$ silicon wafer was oxidized with an oxide layer of $1 \mu \mathrm{m}$ thickness on both sides, which could function as a buffer layer to circumvent the interaction of catalyst particles with the silicon during CNT growth process. Then a layer of Fe catalyst was deposited onto its top surface with the nominal thickness of $2 \mathrm{~nm}$ by electron beam evaporation. After slicing the wafer into small samples, the sample was kept in the CVD quartz chamber for CNT growth.

4.2. Growth Process. In these experiments, we set the initial temperature ramping rate as $50^{\circ} \mathrm{C} / \mathrm{min}$ and the final $\mathrm{CNT}$ growth temperature $T G=725^{\circ} \mathrm{C}$ in the control program, and the pressure inside the chamber was maintained at about 11 Torr. Based on the temperature profile of the substrate as shown in Figure 2(A), the growth could be divided into 3 steps. Similar to our previous CVD processes $[16,17,19]$, the gas mixture of hydrogen $\left(\mathrm{H}_{2}\right)$ and argon (Ar) was provided throughout the 3 steps: Ar functions as the carrier gas and helps to dilute the acetylene $\left(\mathrm{C}_{2} \mathrm{H}_{2}\right)$ concentration, while $\mathrm{H}_{2}$ acts as the reductive agent to refresh the activity of catalyst particles during the growth [20]; the carbon source $\mathrm{C}_{2} \mathrm{H}_{2}$ was only introduced in the second step to initiate and maintain the CNT growth. The gas flow rate was controlled and monitored in situ with mass flow controller.

To begin with (starting from time $t=t_{0}$ ), the temperature $T$ increases dramatically from initial temperature $T_{0}$ (usually its room temperature) towards the growth temperature $T_{G}$ $\left(725^{\circ} \mathrm{C}\right.$ here). The temperature ramping rate is not constant with time; instead it decreases as $T$ is approaching $T_{G}$.

When the temperature reached $T_{G}\left(t_{1}\right)$, the second step began. The catalyst layer went through some minutes of pretreatment (annealing), so as to further turn its thin film morphology into isolated small particles by increasing surface tension. Then at time $t_{2}$, the $\mathrm{C}_{2} \mathrm{H}_{2}$ gas was introduced into the chamber to initiate the CNT growth. After 30 minutes of growth, $\mathrm{C}_{2} \mathrm{H}_{2}$ supply was stopped at $t_{3}$, and the system started to cool down until below $200^{\circ} \mathrm{C}$, when the samples could be taken out for characterizations. The surface morphology and the length of the as-grown CNTs on substrate were characterized with scanning electron microscopy (SEM), Hitachi S-3500 N. Typical SEM image of the CNT mat is shown in Figure 2(B)(a), the as-grown CNT mat is perpendicular to the substrate top surface, with uniform thickness of about $320 \mu \mathrm{m}$, and the closer look in Figure 2(B)(b) reveals that these CNTs are densely packed with a bit wavy entanglement between them. The transmission electron microscopy (TEM, model: FEI Titan) is used to characterize the structure of CNTs with very high resolution. As shown in Figure 2(B)(c)-(d), the CNTs grown here are multiwall carbon nanotubes (MWCNTs) with 10-30 walls and $10-30 \mathrm{~nm}$ in outermost diameter.

\section{Modeling Results and Discussions}

In this study, five input patterns were used during the CNT growth (values of $\mathrm{C}_{2} \mathrm{H}_{2}, \mathrm{Ar}, \mathrm{H}_{2}$, pretreatment, and growth duration) and length of CNT is considered as the only output. The set of training data is comprised of $90 \%$ experimental 


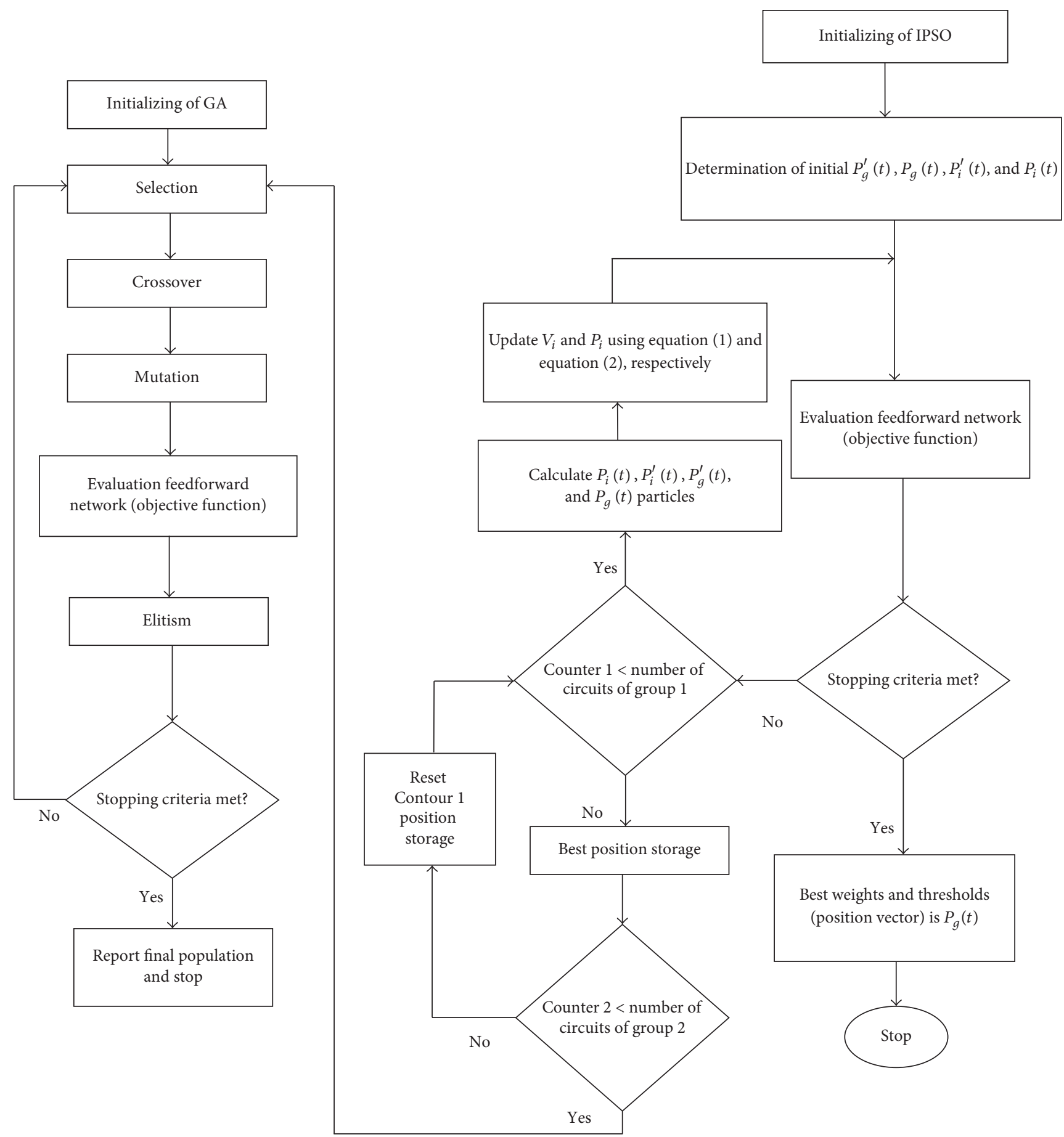

FIGURE 1: Flow chart of the proposed neural network trained by hybrid PSO and GA algorithms.

data (43 groups). 10\% (5 groups) of data was randomly set aside for testing purposes. Stop criteria were selected with either 1500 as iterations or 0.005 as minimum error (MSE) condition for all networks. A preliminary analysis was performed on conventional NNs which has not been presented in this paper to establish a control for comparison with the proposed PSO ANN. The LM-NN performed best in both the training and test data sets out of traditional NNs, yielding the lower mean-square-error, MSE. Tables 1 and 2 compare the performance of CPSONN LM-NN and IPSOGANN. In both training and testing phases IPSOGANN proved superior to CPSONN and LM-NN. The data suggests IPSOGANN can provide a 55\% (MSE) improvement over CPSONN and 80\% (MSE) improvement over LM-NN. During the training it is also found that in terms of speed of convergence (number of needed iterations to meet the stop criteria) PSONNGA is approximately $80 \%$ faster as compared to CPSONN (see Figure 3). 


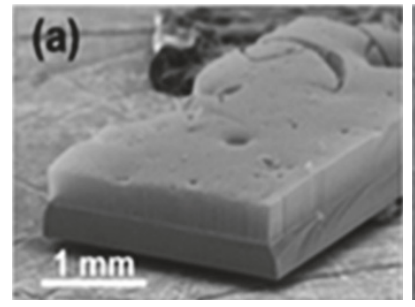

(a)

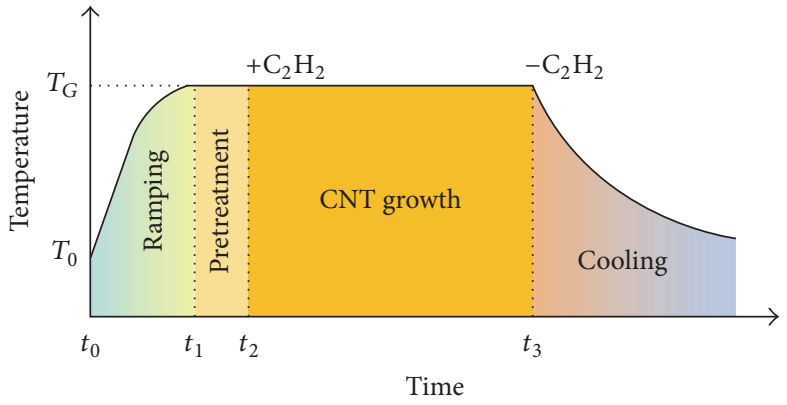

(A)

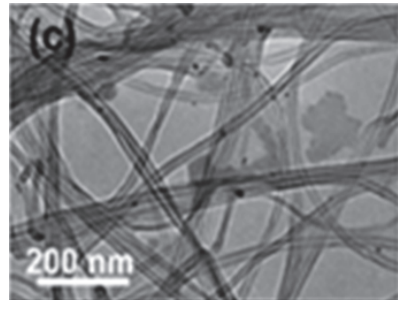

(c)
(B)

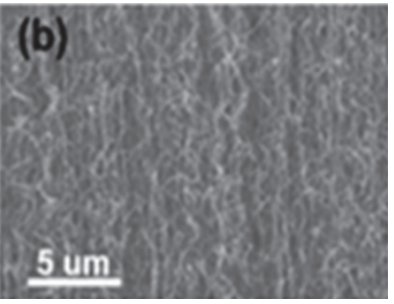

(b)

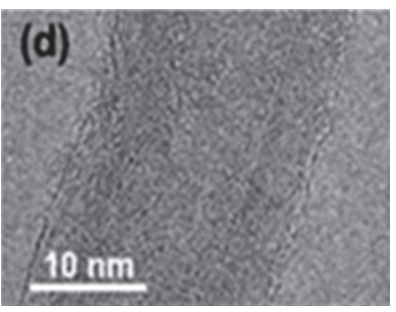

(d)

FIGURE 2: CNT growth process plot. (a) Schematics of the substrate temperature profile during the growth of CNTs. ((b) and (c)) SEM images of grown CNTs. (d) The coaxial walls of a single multiwalled CNT.

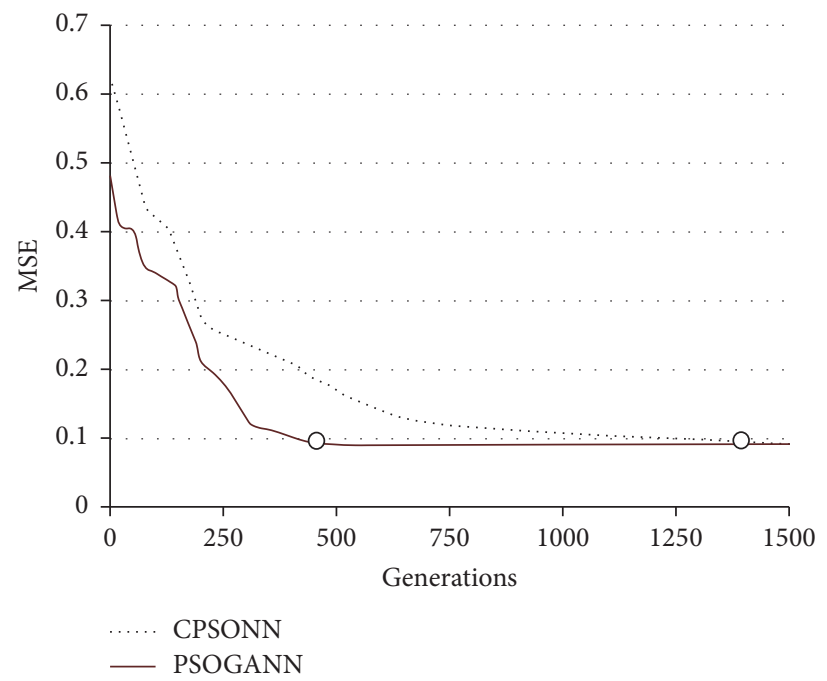

FIGURE 3: Convergence speed for PSONN and PSOGANN algorithms.

TABLE 1: Optimized parameters for LM-NN, CPSONN, and PSOGANN parameters.

\begin{tabular}{lccccccc}
\hline & $V_{\max }$ & $V_{\min }$ & $C_{1}$ & $C_{2}$ & $W$ & Number of particles & Architecture \\
\hline LM-NN & - & - & - & - & - & $5-6-3-1$ \\
CPSONN & 6 & -6 & 2.0 & 1.9 & 0.9 & 30 & $5-4-4-1$ \\
PSOGANN & 7 & -5 & 1.5 & 2.1 & 0.9 & 30 & $5-7-3-1$ \\
\hline
\end{tabular}

TABLE 2: Comparison between LM-NN, CPSONN, and IPSONN for (a) Model I and (b) Model II.

\begin{tabular}{|c|c|c|c|c|c|c|}
\hline & \multicolumn{2}{|c|}{ LM-NN } & \multicolumn{2}{|c|}{ CPSONN } & \multicolumn{2}{|c|}{ PSOGANN } \\
\hline & Train & Test & Train & Test & Train & Test \\
\hline MSE (m2) & 0.244 & 0.269 & 0.091 & 0.106 & 0.041 & 0.057 \\
\hline$R^{2}$ & 0.760 & 0.789 & 0.895 & 0.873 & 0.918 & 0.902 \\
\hline NS & 0.712 & 0.745 & 0.807 & 0.778 & 0.886 & 0.854 \\
\hline
\end{tabular}




\section{Conclusion}

This study proposed a novel algorithm based on PSO and GA for training ANNs (PSOGANN). Application of the proposed algorithm for modeling growth of CNTs is discussed. In particular, proposed model has demonstrated about $40 \%$ improvement in offline training average error in comparison to those of conventional PSO-based ANN algorithm. PSOGANN can be trained extremely quickly, which makes it possible to perform a large number of evaluations required by GA. This method is less sensitive to the permutation problem and improves the results of the evolved networks. This method can highly solve some critical issues associated with traditional neural network systems such as overfitting and falling in local minimum.

\section{Conflicts of Interest}

The authors declare that there are no conflicts of interest regarding the publication of this paper.

\section{Acknowledgments}

Majid Ebrahimi Warkiani would like to acknowledge the support of the Australian Research Council through a Discovery Project Grant (DP170103704).

\section{References}

[1] K. Bosnick, S. Ban, W. Hiebert et al., "Organic vapor adsorption on in situ grown carbon nanotube films," Carbon, vol. 49, no. 11, pp. 3639-3644, 2011.

[2] H. C. Prasad, S. A. R. Hashmi, A. Naik, and H. N. Bhargaw, "Improved shape memory effects in multiwalled-carbon-nanotube reinforced thermosetting polyurethane composites," Journal of Applied Polymer Science, vol. 134, no. 7, Article ID 44389, 2017.

[3] R. M. Ton, D. Munteanu, and G. C. Cocina, "Concept of artificial neural network (ANN) and its application in cerebral aneurism with multi walls carbon nanotubes (MWCNT)," in Proceedings of The 10th Wseas International Conference on Neural Networks, 2009.

[4] S. Yamacli and M. Avci, "Neural network modeling of voltagedependent resistance of metallic carbon nanotube interconnects: An ab initio study," Expert Systems with Applications, vol. 37, no. 12, pp. 8014-8018, 2010.

[5] M. Zarei, A. R. Khataee, R. Ordikhani-Seyedlar, and M. Fathinia, "Photoelectro-Fenton combined with photocatalytic process for degradation of an azo dye using supported $\mathrm{TiO}_{2}$ nanoparticles and carbon nanotube cathode: neural network modeling," Electrochimica Acta, vol. 55, no. 24, pp. 7259-7265, 2010.

[6] M. Zarei, A. Niaei, D. Salari, and A. R. Khataee, "Removal of four dyes from aqueous medium by the peroxi-coagulation method using carbon nanotube-PTFE cathode and neural network modeling," Journal of Electroanalytical Chemistry, vol. 639, no. 1-2, pp. 167-174, 2010.

[7] M. Farahnakian, M. R. Razfar, M. Moghri, and M. Asadnia, "The selection of milling parameters by the PSO-based neural network modeling method," International Journal of Advanced Manufacturing Technology, vol. 57, no. 1-4, pp. 49-60, 2011.
[8] G. S. Carmantini, P. beim Graben, M. Desroches, and S. Rodrigues, "A modular architecture for transparent computation in recurrent neural networks," Neural Networks, vol. 85, pp. 85-105, 2017.

[9] M. R. Razfar, M. Asadnia, M. Haghshenas, and M. Farahnakian, "Optimum surface roughness prediction in face milling X20Cr13 using particle swarm optimization algorithm," Proceedings of the Institution of Mechanical Engineers, Part B: Journal of Engineering Manufacture, vol. 224, no. 11, pp. 1645$1653,2010$.

[10] A. Sinha, P. Malo, and K. Deb, "Evolutionary algorithm for bilevel optimization using approximations of the lower level optimal solution mapping," European Journal of Operational Research, vol. 257, no. 2, pp. 395-411, 2017.

[11] T. Bhoskar, O. K. Kulkarni, N. K. Kulkarni, S. L. Patekar, G. M. Kakandikar, and V. M. Nandedkar, "Genetic Algorithm and its Applications to Mechanical Engineering: A Review," pp. 26242630.

[12] M. Asadnia, L. H. C. Chua, X. S. Qin, and A. Talei, "Improved particle swarm optimization-based artificial neural network for Rainfall-Runoff modeling," Journal of Hydrologic Engineering, vol. 19, no. 7, pp. 1320-1329, 2014.

[13] M. R. Soleymani Yazdi, M. R. Razfar, and M. Asadnia, "Using particle swarm optimization based neural network for modeling of thrust force drilling of PA-6/ Nanoclay Nanocomposites," Applied Mechanics and Materials, vol. 34-35, pp. 722-726, 2010.

[14] A. M. Khorasani, M. Asadnia, and P. Saadatkia, "Modeling of TiC-N Thin Film Coating Process on Drills Using Particle Swarm Optimization Algorithm," Arabian Journal for Science and Engineering, vol. 38, no. 6, pp. 1565-1571, 2013.

[15] G. Das, P. K. Pattnaik, and S. K. Padhy, "Artificial Neural Network trained by Particle Swarm Optimization for non-linear channel equalization," Expert Systems with Applications, vol. 41, no. 7, pp. 3491-3496, 2014.

[16] T. Xu, J. Miao, H. Li, and Z. Wang, "Local synthesis of aligned carbon nanotube bundle arrays by using integrated microheaters for interconnect applications," Nanotechnology, vol. 20, no. 29, Article ID 295303, 2009.

[17] T. Xu, Z. Wang, J. Miao, X. Chen, and C. M. Tan, "Aligned carbon nanotubes for through-wafer interconnects," Applied Physics Letters, vol. 91, no. 4, Article ID 042108, 2007.

[18] M. R. Soleymani Yazdi, M. R. Razfar, and M. Asadnia, "Modelling of the thrust force of the drilling operation on PA6nanoclay nanocomposites using particle swarm optimization," Proceedings of the Institution of Mechanical Engineers, Part B: Journal of Engineering Manufacture, vol. 225, no. 10, pp. 17571771, 2011.

[19] J. Lu, J. Miao, T. Xu, B. Yan, T. Yu, and Z. Shen, "Erratum: Growth of horizontally aligned dense carbon nanotubes from trench sidewalls (Nanotechnology (2011) 22 (265614))," Nanotechnology, vol. 22, no. 47, Article ID 479502, 2011.

[20] F. Ohashi, G. Y. Chen, V. Stolojan, and S. R. P. Silva, "The role of the gas species on the formation of carbon nanotubes during thermal chemical vapour deposition," Nanotechnology, vol. 19, no. 44, Article ID 445605, 2008. 

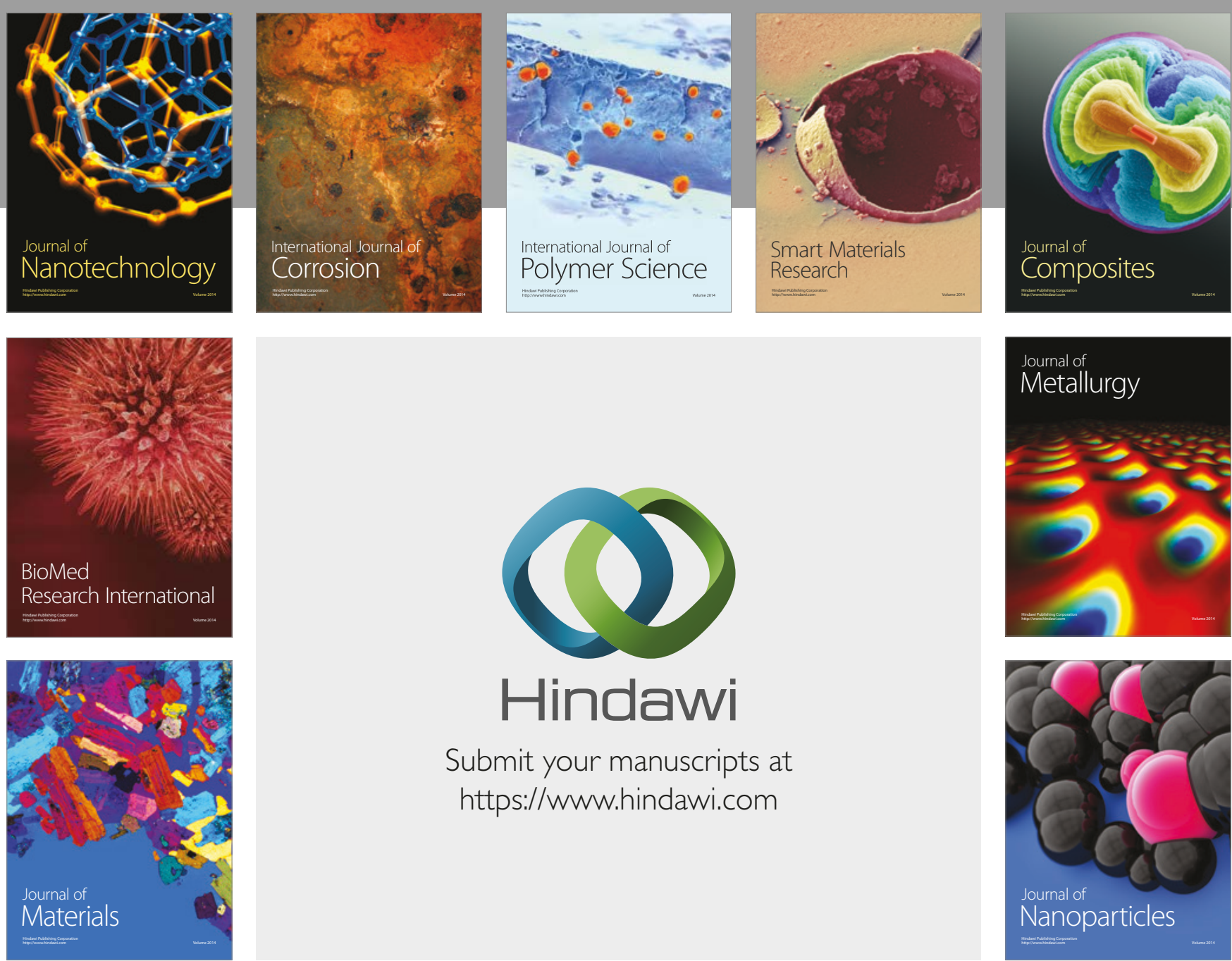

\section{Hindawi}

Submit your manuscripts at

https://www.hindawi.com
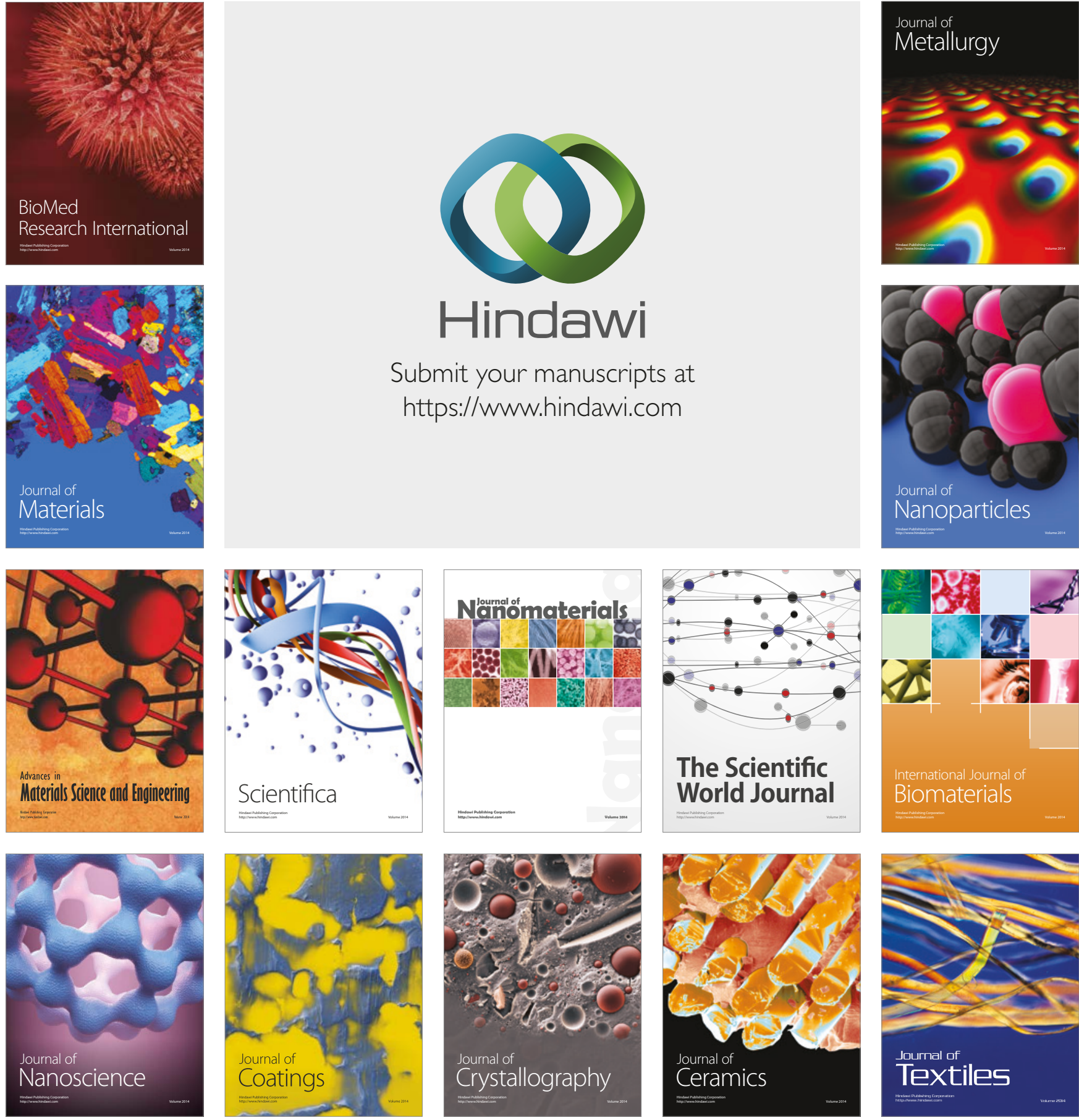

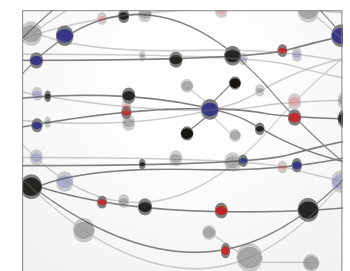

The Scientific World Journal
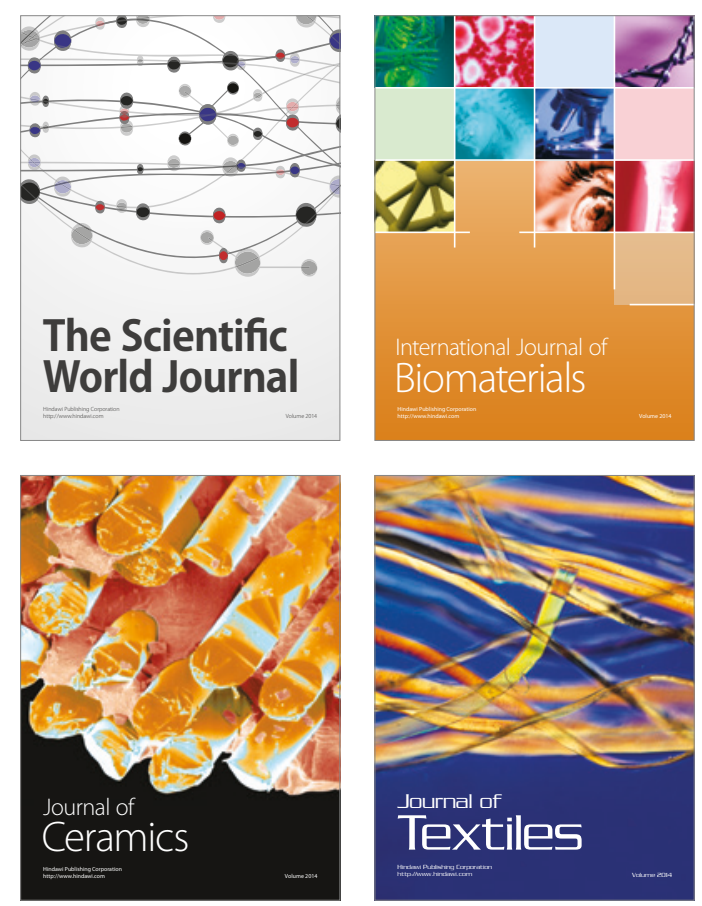